\title{
Da política à ação institucional: prioridades de pesquisa no Ministério da Saúde
}

\section{From political to institutional action: research priorities in the Ministry of Health}

Decit - Departamento de Ciência e Tecnologia do Ministério da Saúde Esplanada dos Ministérios

Bloco G sala 845

70058-900 Brasília, DF, Brasil

Texto de difusão técnico-científica do Ministério de Saúde.

\author{
Departamento de Ciência e Tecnologia. Secretaria de Ciência e \\ Tecnologia e Insumos Estratégicos do Ministério da Saúde
}

Conjugar a saúde pública ao vasto campo da ciência, tecnologia e inovação é avançar no caminho do desenvolvimento econômico e do progresso social de uma nação. Para que o Brasil alcance esse horizonte, voltado às reais necessidades da população, é fundamental abreviar o hiato entre os diversos redutos de pesquisa acadêmica e institucional e os de gestão pública. Assim, aproxima as atividades científicas às ações de prevenção e controle dos problemas de saúde que mais acometem a sociedade, como as recorrentes doenças emergentes e negligenciadas que abatem os países em desenvolvimento.

A evolução das atividades de fomento nessa área no Brasil atesta o progressivo empenho político do Poder Público em seguir a assertiva da Organização Mundial da Saúde (OMS): "geração de novos conhecimentos por meio do método científico para identificar e lidar com problemas de saúde" (Relatório da Comissão sobre Pesquisas em Saúde para o Desenvolvimento - 1990).

Paralela à orientação internacionalmente consolidada, e aprimorada ao longo dos anos, um novo projeto nacional se desenha, com a Política Nacional de Ciência, Tecnologia e Inovação em Saúde (PNCTIS) e a implementação da Agenda Nacional de Prioridades de Pesquisa em Saúde (ANPPS). Essas iniciativas foram inscritas no momento histórico da $2^{\mathrm{a}}$ Conferência Nacional Ciência, Tecnologia e Inovação em Saúde, ocorrida em 2004, que sublinhou o pacto entre usuários, trabalhadores, gestores e pesquisadores em torno da indução das pesquisas segundo as linhas mestras da ANPPS.

Esses instrumentos, implementados pelo Departa- mento de Ciência e Tecnologia do Ministério da Saúde (Decit-MS) e o Conselho Nacional de Saúde, foram somados às parcerias com a Unesco e o Ministério da Ciência e Tecnologia (MCT) - por meio do Conselho Nacional de Desenvolvimento Científico e Tecnológico (CNPq) e a Financiadora de Estudos e Projetos (FINEP). Isso resultou no lançamento de 30 editais temáticos e 1.277 projetos financiados, de 2003 a 2005.

Nos últimos três anos, as ações de fomento têm merecido atenção política e recursos crescentes. A expansão orçamentária é evidente: enquanto em 2003 investiu-se $\mathrm{R} \$ 6,7$ milhões em 151 projetos de pesquisa, em 2004, 844 projetos foram financiados com R\$60,2 milhões. Destes, 441 projetos são voltados ao desenvolvimento regional integrando o Programa Pesquisa para o SUS (PPSUS): gestão compartilhada em saúde, somando R $\$ 14,6$ milhões. Em 2005, o montante de $\mathrm{R} \$ 127,8$ milhões foi investido pelos parceiros do MS, como CNPq, Finep e Unesco. Foram financiados 416 projetos por meio de editais temáticos e pesquisas estratégicas e de desenvolvimento tecnológico no complexo produtivo da saúde. Ainda nesse ano, em cooperação técnica com a Finep, foram dedicados $\mathrm{R} \$ 72,1$ milhões em 38 pesquisas relacionadas aos editais Rede Multicêntrica de Avaliação de Implantes Ortopédicos (Remato), Implantação de Unidades de Pesquisa Clínica em Hospitais de Ensino, Estudo Multicêntrico Longitudinal em Doenças Cardiovasculares e Diabetes Mellitus (ELSA). E por meio de convênio com a Unesco, a Pesquisa Nacional de Demografia e Saúde da Criança e da Mulher (PNDS) foi financiada com $\mathrm{R} \$ 7,7$ milhões.

A Figura 1 mostra a evolução das ações de fomento 


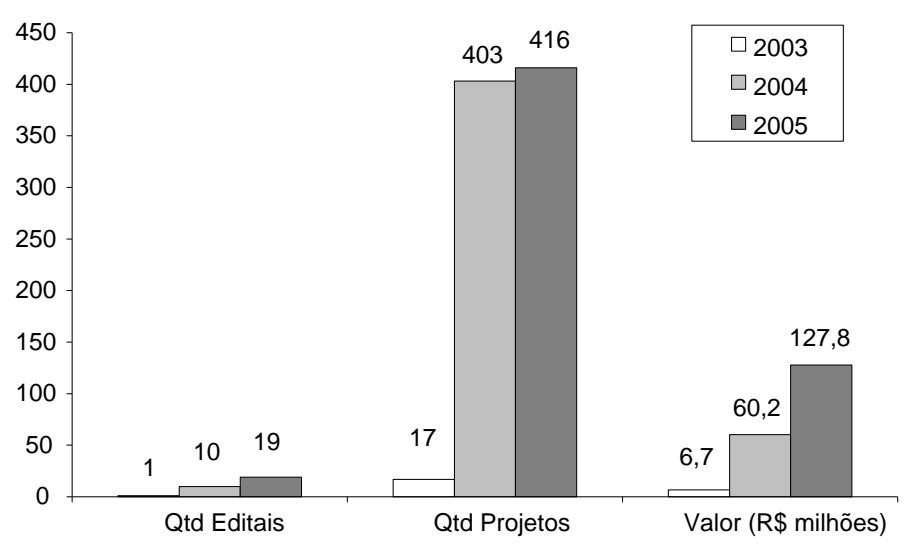

*Projetos apoiados e valores financiados em cooperação técnica com o CN Pq, Finep, Unesco e Fundo Nacional de Saúde (FNS).

Fonte: Departamento de Ciência e Tecnologia do Ministério da Saúde (Decit) lume financeiro executado compreende projetos selecionados por meio de chamadas públicas, seguindo os critérios de mérito científico e relevância para a saúde pública. $\mathrm{Na}$ Figura 2, observa-se a série histórica de incentivo à pesquisa entre os anos $2003 \mathrm{e}$ 2005 , com a evolução da modalidade de fomento por meio de editais temáticos e chamadas públicas. As contratações por encomenda - que em 2003 consumiam 55,3\% dos recursos do Decit - diminuíram significativamente para o patamar de apenas $11,1 \% \mathrm{em}$ 2005. A ilustração confirma, assim, a consolidação de uma política de eqüidade e de participação democrática no incentivo às atividades de pesquisa.

Em 2006, o Ministério da Saúde investirá no período de 2003 a 2005, em número de editais lançados, quantidade de projetos e valor de recursos financeiros empregados. É possível perceber um aumento significativo de todas as variáveis no ano de 2005.

Em 2004 e 2005, foram apoiados 1.260 estudos em diversas áreas do conhecimento: alimentação e nutrição, violência, acidentes e trauma, sistemas e políticas em saúde, mortalidade materna e morbi-mortalidade neonatal, saúde bucal, hantavírus e outras retroviroses, saúde mental, saúde dos povos indígenas, neoplasias, terapia celular clínica e pré-clínica, avaliação econômica e análise de custos, hanseníase, PPSUS Amazônia, bioética, avaliação do Sistema CEP (Comitê de Ética em Pesquisa)/Conep (Comissão Nacional de Ética em Pesquisa), Baixada Santista, BR 163 (pavimentação da BR e impactos na saúde) e assistência farmacêutica.

Orientada pela premissa ética da responsabilidade pública, a política de alocação dos recursos do Decit é pautada de forma transparente, em que o maior vo- mais $\mathrm{R} \$ 125,9$ milhões em pesquisas selecionadas por meio de editais temáticos - quatro em parceria com a Finep e oito com o CNPq (Tabela). Essas chamadas públicas foram constituídas a partir do debate entre representantes da gestão pública, da comunidade científica e acadêmica, e dos usuários.

Um dos eixos estratégicos inscritos na PNCTIS é a difusão das informações técnico-científicas em saúde - instância fundamental para assegurar o controle social e a transparência dos investimentos governamentais. Encontra-se em fase de implantação o Observatório de Ciência, Tecnologia e Inovação em Saúde, uma iniciativa do Decit com a cooperação técnica da Fiocruz. O objetivo é constituir um instrumento de monitoramento das ações de fomento, que irá conferir visibilidade pública às pesquisas financiadas pelo Ministério da Saúde.

A política adotada pelo Decit de incentivar o desenvolvimento regional apresentou grande efetividade no ano de 2005: do total de recursos empenhados, $31,5 \%$ foram destinados às regiões Norte, Nordeste $\mathrm{e}$

Tabela - Editais do Decit em parceria com a FINEP e o CNPq, 2006.

\begin{tabular}{lr}
\hline Editais & Valor (R\$) \\
\hline Avaliação Tecnológica em Saúde (ATS)/REBRATS & $7.857 .000,00$ \\
Inovação em Produtos Terapêuticos e Diagnósticos & $63.570 .000,00$ \\
Subtotal FIN EP & $71.440 .000,00$ \\
Doenças N egligenciadas & $20.000 .000,00$ \\
Envelhecimento Populacional e Saúde da Pessoa Idosa & $6.000 .000,00$ \\
Determinantes Sociais e Populações Vulneráveis (população masculina, com deficiência e pessoas idosas) & $10.000 .000,00$ \\
Saúde e Ambiente & $3.500 .000,00$ \\
Gestão do Trabalho em Saúde; Gestão da Educação em Saúde; Comunicação e Informação em Saúde & $6.000 .000,00$ \\
Potencial Farmacológico de Organismos Marinhos & $3.000 .000,00$ \\
Genética Clínica & $4.000 .000,00$ \\
Saúde Suplementar* & $2.000 .000,00$ \\
Subtotal CN Pq & $54.500 .000,00$ \\
\hline Total (FINEP + CN Pq) & $125.940 .000,00$ \\
\hline
\end{tabular}


Centro-Oeste. Esse percentual manteve uma similaridade em todas as parcerias com as agências de fomento (Figura 2).

\section{Percurso democrático}

O processo de construção colegiada dos editais, segundo os marcos da Agenda $\mathrm{Na}-$ cional de Prioridades de Pesquisa, vem sendo aprimorado ano-a-ano. Com o objetivo de consolidar e legitimar a continuidade desse democrático percurso e ampliar as instâncias de interlocução entre gestores, pesquisadores e usuários das esferas nacional, estaduais e municipais, o Decit promoveu em 2006 duas grandes oficinas que reuniram ao

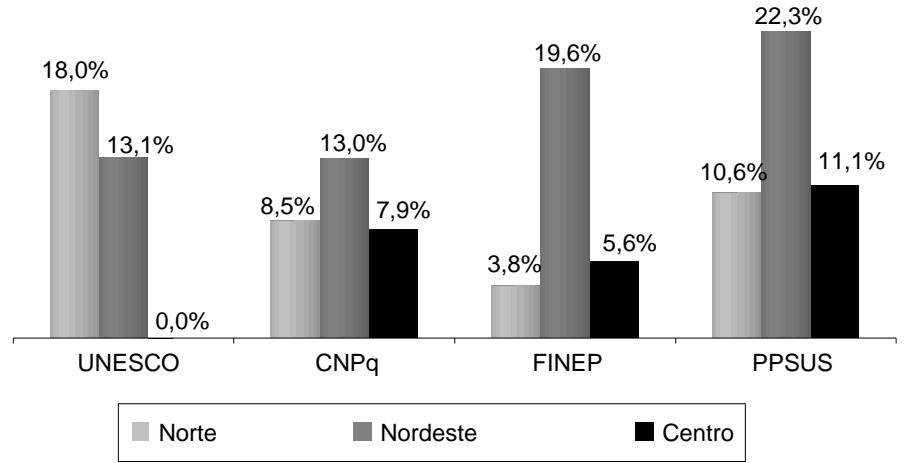

Fonte: Departamento de Ciência e Tecnologia do Ministério da Saúde

Figura 2 - Distribuição percentual dos recursos financeiros para fomento à pesquisa nas regiões Norte, Nordeste e Centro-O este, segundo as agências de financiamento, 2005. todo, entre as principais autoridades em saúde pública do Brasil, cerca de 250 representantes desses segmentos.

A primeira iniciativa, intitulada de Oficina de Prioridades de Pesquisa em Saúde - Editais 2006, ocorrida em março último em Brasília, cumpriu o papel de definição das principais políticas norteadoras dos temas que configuraram as 12 chamadas públicas a serem contempladas nesse ano. A lógica de elaboração dos editais ultrapassa a simples enumeração das enfermidades e agravos, seguindo a complexidade do debate que deriva da constatação dos quadros que apontam a necessidade de aperfeiçoamento das políticas públicas, confirmam as lacunas de conhecimento em diversas áreas de pesquisa e apresentam a disponibilidade de recursos financeiros para o setor.

Foram constituídos grupos com especialistas nas seguintes áreas: Comunicação e Informação em Saúde; Gestão do Trabalho em Saúde; Gestão da Educação em Saúde; Avaliação das Tecnologias em Saúde: Genética Clínica; Saúde da População Negra; Saúde da Pessoa com Deficiência; Saúde da População Masculina; e Fármacos.

Foram também apresentadas as conclusões quanto a temas específicos que também resultaram nos editais de Saúde e Ambiente, Envelhecimento Populacional e Saúde da Pessoa Idosa, Determinantes Sociais da Saúde, e Kits Diagnósticos para Doenças Negligenciadas.

\section{Metodologia}

A definição de prioridades de pesquisa foi pautada pela metodologia da Matriz Combinada, que considera cinco elementos: a magnitude do problema (carga, mortalidade, deficiência, iniqüidade); os fatores de risco (razões pelas quais o problema persiste) como de- terminantes do agravo; o conhecimento atual; o possível impacto da pesquisa; e os fluxos financeiros.*

A seguir, as linhas-mestras que balizaram os editais lançados em 2006:

1. Avaliação de Tecnologias em Saúde (ATS) - Por se tratar de uma discussão específica, o grupo de trabalho adotou uma metodologia diferenciada, que resultou em deliberações pertinentes às necessidades da área, tais como: estruturar a Rede Brasileira de ATS em Saúde (Rebrats); elaborar guias metodológicos; desenvolver sistema de informação de estudos em ATS; articular a Rede Brasileira de Pesquisa Clínica em Hospitais de Ensino com a rede ATS.

2. Envelhecimento Populacional e Saúde da Pessoa Idosa - Linhas prioritárias: funcionalidade e fragilidade na Atenção à Saúde da Pessoa Idosa; modelos interdisciplinares de cuidado ao idoso com condições crônicas.

3. Genética Clínica - Manejo e prevenção das anomalias congênitas; câncer familial; doenças neuromusculares; erros inatos do metabolismo; hemoglobinopatias hereditárias; e retardo mental.

4. Gestão da Educação - Referenciais teórico-metodológicos de projetos curriculares para a formação profissional em saúde; avaliação dos processos de mudança na formação profissional e do impacto da participação docente e discente na mudança das práticas de saúde; avaliação dos programas de pós-graduação lato sensu apoiados pelo MS

5. Gestão do Trabalho em Saúde - Adequação dos mecanismos de gestão do trabalho às ações e serviços no SUS: metodologias para o dimensionamento de força de trabalho; avaliação dos mecanismos de incentivo à produtividade e de avaliação de desempenho; análise de custos com a for- 
ça de trabalho em saúde. Quanto à regulação do trabalho são necessárias pesquisas abordando: 0 impacto da Lei de Responsabilidade Fiscal e a legislação referente à reforma administrativa do Estado no processo de precarização/desprecarização; e as modalidades de contratação/vínculos nos diversos níveis de complexidade do SUS.

6. Informação e Comunicação em Saúde - Gestão e uso da informação na saúde; avaliação; desenvolvimento tecnológico; qualidade.

7. Kits Diagnósticos - Pesquisa, desenvolvimento e inovação de produtos diagnósticos em saúde. Como eixos prioritários foram definidas as doenças negligenciadas ou de impacto econômico para o SUS.

8. Saúde da Pessoa com Deficiência - Avaliação dos serviços que integram a rede de atenção à saúde de pessoas com deficiência física, auditiva, visual e mental, em todos os níveis de atenção; do acesso e a acessibilidade aos serviços; da efetividade da reabilitação e no controle das doenças infecto-contagiosas na infância; do custo $v s$ efetividade da triagem auditiva neonatal em maternidades do SUS; do uso das ajudas técnicas relacionadas ao cotidiano. Formular indicadores de vulnerabilidade à saúde utilizando como referência a Classificação Internacional de Funcionalidade, Incapacidade e Saúde; avaliar medidas especiais de proteção à saúde do trabalhador admitido pelo sistema de cotas e os recursos tecnológicos na reabilitação motora do indivíduo amputado; avaliar a efetividade das intervenções precoces no acidente vascular encefálico e no trauma crâneo-encefálico e raqui-medular.

9. Saúde da População Masculina - Estudos de determinantes da carga da doença/agravos predominantes na população masculina e seus fatores de risco, fisiopatologia e aspectos clínicos; avaliação da atenção à saúde da população masculina; sexualidade e saúde reprodutiva; saúde e trabalho; desenvolvimento, aplicação e avaliação de novas tecnologias de atenção à saúde da população masculina.

10. Saúde da População Negra - Racismo como determinante social das condições de saúde; racismo e saúde mental; doença falciforme; territórios promotores de saúde; epidemiologia e controle das doenças e agravos prevalentes; avaliação de políticas, programas e ações; e mobilização, participação e controle social.

11.Determinantes Sociais - Desigualdades sociais, regionais, étnico-raciais e de gênero - morbidade e fatores de risco; desigualdades no acesso e qualidade dos serviços de saúde e intervenções sociais; aspectos metodológicos dos estudos sobre determinantes sociais de saúde.
12. Fármacos - Inovação na cadeia tecnológica e industrial na área farmacêutica, especialmente formulação farmacêutica, transposição de escala, testes pré-clínicos, toxicológicos e ensaios clínicos.

13. Saúde e Ambiente - Saúde de populações expostas a contaminantes químicos em áreas com solo contaminado.

\section{Doenças negligenciadas}

Prosseguindo com o processo de busca da metodologia mais adequada para a composição dos editais de pesquisa, o Decit, em parceria com a Fundação Oswaldo Cruz (Fiocruz), realizou outro fórum de discussão: a Oficina de Prioridades de Pesquisa em Doenças Negligenciadas com o objetivo de estabelecer as linhas prioritárias para conduzir os estudos relacionados à dengue, doença de chagas, hanseníase, leishmaniose, malária e tuberculose. $\mathrm{O}$ evento, que reuniu especialistas nos estudos dessas patologias, contou com a participação do ministro interino da Saúde, e foi coordenado pelo diretor do Centro de Desenvolvimento Tecnológico em Saúde da Fiocruz, o professor Carlos Morel. Com reconhecida experiência nacional e internacional em doenças negligenciadas, especialmente as tropicais, Morel esteve à frente do grupo Special Program for Research and Training in Tropical Diseases (TDR), ligado à OMS.

Ao todo foram 70 pessoas, entre as principais autoridades nas áreas, gestores de saúde e pesquisadores de todas as regiões do Brasil, que discutiram, além das questões específicas de cada edital, o papel da indústria farmacêutica e dos governos dos países desenvolvidos no enfrentamento dessas enfermidades que acometem principalmente as camadas mais pobres da população.

Uma constatação fundamental ressaltada no debate é a necessidade de enfrentamento a falhas em três campos: da ciência, do mercado e da saúde pública. Essa análise faz-se imprescindível para orientar a sistematização de prioridades nos projetos a serem financiados.

Com financiamento de $\mathrm{R} \$ 20$ milhões previsto para este ano, as prioridades elencadas no edital de Doenças Negligenciadas são:

- Tuberculose - Indicadores de serviços de saúde; parâmetros da dinâmica da tuberculose e sua aplicação na vigilância epidemiológica; distribuição espacial e caracterização de áreas de risco; contatos de tuberculose pulmonar e mecanismos de transmissão e medidas controle; eficácia e efetividade de novos medicamentos, esquemas e regimes terapêuticos e profiláticos.

- Malária - Estudos clínicos e pré-clínicos; identi- 
ficação de novos alvos para vacinas, diagnóstico, imunoterapia, a partir das tecnologias de genoma e pós-genoma; mecanismos moleculares de resistência de parasitas a drogas e de vetores a inseticidas e identificação de novos alvos e desenvolvimento de novos produtos, estudos de fisiopatologia; estudos epidemiológicos sobre a prevalência e papel de portadores assintomáticos de parasita e vetores e proposição de métodos alternativos de controle.

- Doença de Chagas - Estudo de vetores; identificação de novas drogas e aperfeiçoamento da quimioterapia; identificação de marcadores de evolução e prognóstico da doença de Chagas crônica e de resistência do Trypanosoma cruzi a drogas.

- Hanseníase - Novos testes para diagnóstico, resistência, suscetibilidade e transmissão; marcadores preditivos de reação hansênica; marcadores de grupos de risco; novos esquemas e regimes para o tratamento a hanseníase e reações hansênicas; avaliação das estratégias para o controle da hanseníase na atenção básica à saúde.

- Leishmaniose - Estudos do parasita; estudo das doenças. Diagnóstico; tratamento; vacinação; vetores; reservatórios e controle.

- Dengue - Estudo de vetores; estudo de epidemiologia; vigilância e controle; e estudos clínicolaboratoriais. 